Natalia Wiktorczyk ${ }^{1}$, Katarzyna Grudlewska ${ }^{1}$, Krzysztof Skowron¹, Grzegorz Gryń2, Eugenia GospodarekKomkowska ${ }^{1}$

${ }^{1}$ Department of Microbiology, Nicolaus Copernicus University in Toruń, Collegium Medicum of L. Rydygier in Bydgoszcz, Bydgoszcz, Poland 2Plant Breeding and Acclimatization Institute - National Research Institute, Bydgoszcz, Poland

\title{
The effect of blood on the ability of biofilm formation by Listeria monocytogenes strains
}

\author{
Corresponding author: \\ Natalia Wiktorczyk, MSc \\ Department of Microbiology, Nicolaus \\ Copernicus University in Toruń, \\ Collegium Medicum of L. Rydygier \\ in Bydgoszcz \\ 9 M. Skłodowskiej-Curie St \\ 85-094 Bydgoszcz, Poland \\ e-mail: n.wiktorczyk@cm.umk.p \\ Tel.: +48667378325 \\ Fax: +48525854080
}

\begin{abstract}
Introduction: Listeria monocytogenes strains are widespread in the natural environment and are the etiological factor of listeriosis. Food is the main source of $L$. monocytogenes. Secondary contamination of food products which results from the formation of biofilm by L. monocytogenes on the surfaces of the processing devices makes an essential problem.

Material and methods: We evaluated the ability of biofilm formation in media supplemented with sheep blood (in a volume of 5.0, 10.0, 20.0 and 50.0\%) for the reference strain L. monocytogenes ATCC 7644 and strains isolated from the human blood and carrots on the polypropylene surface.

Results: The strain isolated from blood most efficiently formed a biofilm with increasing blood sheep's addition (nutrient broth $-4.87 \times 10^{5} \mathrm{CFU} \times \mathrm{cm}^{-2}$, blood supplement $50.0 \%(\mathrm{v} / \mathrm{v})-3.4 \times 10^{7} \mathrm{CFU} \times$ $\left.\mathrm{cm}^{-2}\right)$. The number of $L$. monocytogenes recovered from the biofilm for the reference strain and the strain from carrot increased to $20.0 \%$ by volume of the blood addition $\left(3.07 \times 10^{5} \mathrm{CFU} \times \mathrm{cm}^{-2}\right.$ and $4.03 \times 10^{5}$ $\mathrm{CFU} \times \mathrm{cm}^{-2}$ respectively - nutrient broth; $1.1 \times 10^{7} \mathrm{CFU} \times \mathrm{cm}^{-2}$ and $9.23 \times 10^{6} \mathrm{CFU} \times \mathrm{cm}^{-2}-$ blood supplement $20.0 \%(\mathrm{v} / \mathrm{v})$ ). Decrease in the number of cells recovered from the biofilm for the reference strain and the one isolated from carrot has been demonstrated at $50.0 \%$ addition of blood into nutrient medium $\left(8.8 \times 10^{6} \mathrm{CFU} \times \mathrm{cm}^{-2}\right.$ and $7.87 \times 10^{6} \mathrm{CFU} \times \mathrm{cm}^{-2}$ respectively).

Conclusion: The addition of sheep blood to the medium at concentrations up to $20.0 \%$ increases the number of cells recovered from the biofilm for all studied $L$. monocytogenes strains whereas medium with $50.0 \%$ blood stimulates the biofilm formation only by the strain isolated from blood. Key words: biofilm, Listeria monocytogenes, nutrient broth, blood, polypropylene
\end{abstract}

Med Res J 2018; 3 (1): 28-31

\section{Introduction}

Listeria monocytogenes are gram-positive, relatively anaerobic, non-enveloped and non-spore-forming bacilli, commonly found in the natural environment (water, soil, sewage, decaying vegetation or silage) [1]. These bacteria cause listeriosis, dangerous for pregnant women and newborns as well as elderly people [2]. Food, including raw meat, fish (fresh and smoked), vegetables, fruits and dairy products are their main source [3]. Secondary contamination of food products may be the result of biofilm formation by $L$. monocytogenes on the surfaces of equipment used in food processing plants [4-5]. L. monocytogenes strains are capable of colonizing various surfaces contacting with food, including polystyrene, polypropylene, glass, stainless steel, quartz, marble and granite [6]. Biofilm formation is induced by many environmental factors found in the food processing industry, such as: $\mathrm{pH}$ changes, increased oxygen concentration, and also depends on the availability of nutrients

[7-8]. Limited access to nutrients induces stress in L. monocytogenes, resulting in increased biofilm formation [7-9]. It has been shown that the presence of food production by-products (meat juice, pork serum or fat) in the processing environment stimulates the biofilm formation [10]. Conversion of planktonic forms to the mature biofilm structure increases the chance of survival of $L$. monocytogenes and determines the acquisition of resistance to environmental factors (UV radiation, 
acids) and antimicrobial compounds, including antibiotics, which makes it difficult to eliminate biofilms in the food processing plants [7-8]. Extracellular polymeric substances (EPS) protect the cells of bacteria in the biofilm from the unfavorable factors of the processing environment. EPS is composed of protein, DNA, polysaccharides and other extracellular compounds. EPS mediates attachment of $L$. monocytogenes to the surface and provides mechanical stability to the formed biofilm [6].

The aim of study was to assess the ability of biofilm formation by $L$. monocytogenes strains on the polypropylene surface in a nutrient medium enriched with sheep blood.

\section{Materials and methods}

\section{Material for research}

The reference Listeria monocytogenes ATCC 7644 strain and two strains of these species isolated from human blood and carrots, from the collection of the Department of Microbiology, L. Rydygier Collegium Medicum in Bydgoszcz, were used for the study. Isolates were stored at $-80^{\circ} \mathrm{C}$ in $\mathrm{BHI}$ (Brain Heart Infusion) medium (bioMérieux) with the addition of $15.0 \%$ glycerol.

\section{Strains preparation}

In the first stage culture of deep-frozen strains on Columbia Agar with $5.0 \%$ sheep blood (bioMérieux) was used. After incubation at $37^{\circ} \mathrm{C}$ for 24 hours, a passage of the grown colonies of the tested strains on the same type of substrate was performed. The obtained colonies of $L$. monocytogenes were used in the subsequent studies.

Assessment of biofilm formation by L. monocytogenes on polypropylene surface in nutrient medium enriched with sheep blood

Sterile polypropylene plates were used in the experiments. The culture medium was prepared by mixing sterile nutrient broth (Merck) with sheep blood. The blood supplement was 5.0, 10.0, 20.0 and $50.0 \%$ of the volume $(\mathrm{v} / \mathrm{v})$. The nutrient broth without addition of sheep blood was used as a control.

Suspensions of examined strains (0.5 McFarland standard) were prepared in $3 \mathrm{ml}$ of phosphate-buffered physiological saline (PBS) (Avantor). Then suspensions were centrifuged for 5 minutes (3000 rpm) and the supernatants removed. The previously prepared medium was added to the test tubes with sediment, mixed for
2 minutes and added on the polypropylene plates that were incubated at $37^{\circ} \mathrm{C}$ for 24 hours. After the incubation, the plates were removed and washed with PBS. Plates with the biofilm of $L$. monocytogenes strains were placed in test tubes containing $3 \mathrm{ml}$ of PBS solution and sonicated for 10 minutes $(30 \mathrm{kHz}, 150 \mathrm{~W})$. A series of decimal dilutions in PBS was made and culture was made on Columbia Agar with $5.0 \%$ sheep blood. The experiment was performed in triplicate for each tested strain. After the incubation period ( 24 hours, $\left.37^{\circ} \mathrm{C}\right)$, the grown colonies were counted and expressed as the number of colony forming units per $\mathrm{cm}^{2}\left(\mathrm{CFU} \times \mathrm{cm}^{-2}\right)$.

\section{Statistical analysis}

The results were subjected to statistical analysis with the use of Statistica 12.0 PL (StatSoft). For each strain, the median number of bacteria recovered from the biofilm was calculated in each variant of the experiment. Statistically significant differences between them were evaluated based on the Bonferroni post-hoc test at the significance level of 0.05 .

\section{Results}

All tested strains of $L$. monocytogenes formed a biofilm, both in the medium with and without the addition of sheep blood (Fig. 1).

In the medium without blood addition (control) the number of cells recovered from the biofilm ranged from $3.07 \times 10^{5} \mathrm{CFU} \times \mathrm{cm}^{-2}$, for the reference L. monocytogenes ATCC 7644 strain, to $4.87 \times 10^{5} \mathrm{CFU} \times \mathrm{cm}^{-2}$ for the human blood isolated strain and $4.03 \times 10^{5} \mathrm{CFU} \times \mathrm{cm}^{-2}$ for a carrot isolated strain (Fig. 1). The number of cells recovered for all tested strains, in the medium without the addition of blood did not differ significantly (Fig. 1).

The addition of sheep blood in the volume of 5.0, 10.0 and $20.0 \%$ to the medium, stimulated the tested strains of $L$. monocytogenes to biofilm formation. For each of them, The number of cells recovered from the biofilm was significantly higher at each subsequent blood addition to the medium (Fig. 1).

In a medium with the highest content of blood the number of cells recovered from the biofilm increased from $9.23 \times 10^{6} \mathrm{CFU} \times \mathrm{cm}^{-2}$ for strain derived from carrots and up to $1.40 \times 10^{7} \mathrm{CFU} \times \mathrm{cm}^{-2}$ for the strain isolated from human blood (Fig. 1).

The increase of blood volume in the medium to $50.0 \%$ resulted in a significant decrease in the number of biofilm cells recovered for the reference $L$. monocytogenes strains and the carrot isolates (Fig. 1). Under this condition only the strain derived from the human blood, strongly formed the biofilm $\left(3.40 \times 10^{7} \mathrm{CFU} \times \mathrm{cm}^{-2}\right)$. 


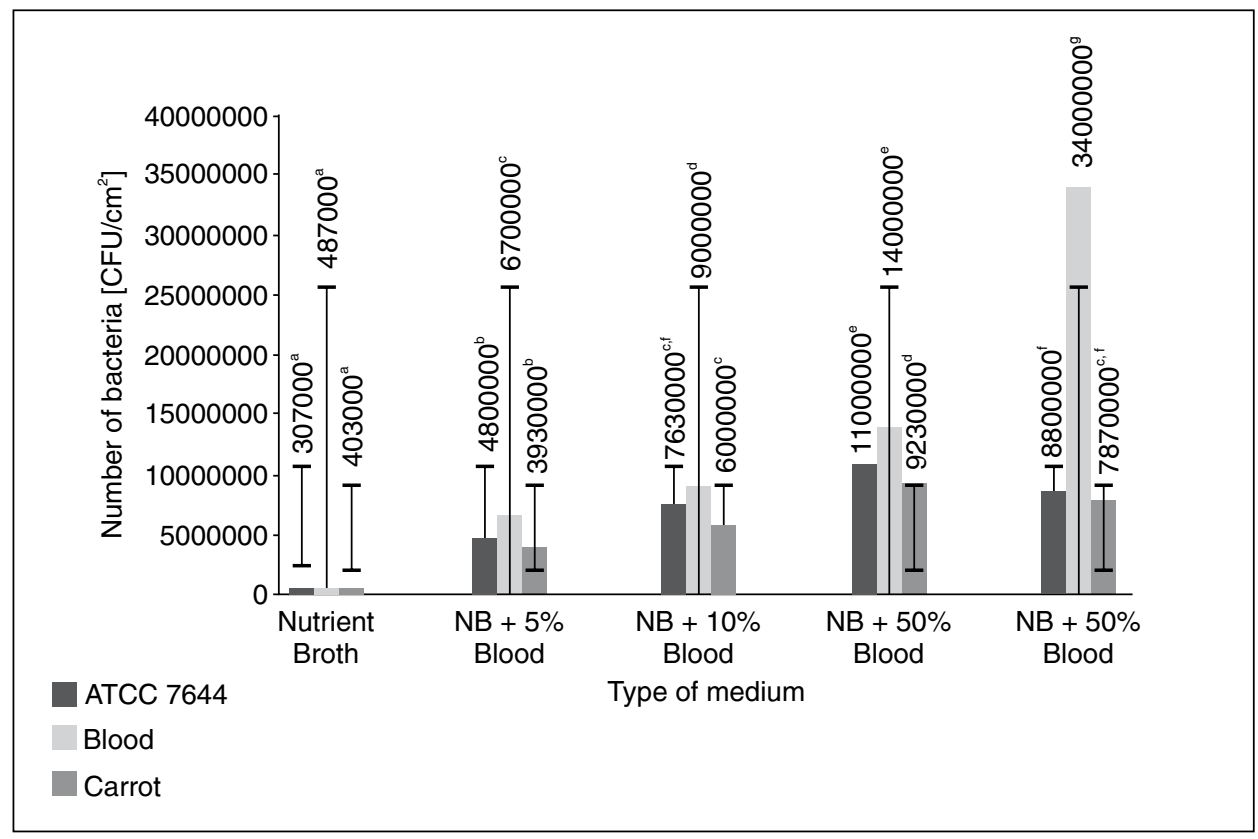

Figure 1. The effect of sheep blood addition on the ability of biofilm formation on the polypropylene surface by L. monocytogenes (ATCC 7644 - reference strain; Blood - L. monocytogenes strain isolated from blood; Carrot - L. monocytogenes strain isolated from carrot). Values marked a, b, c differ significantly.

Regardless of the volumetric addition of blood in the medium, $L$. monocytogenes isolated from blood formed the strongest biofilm whereas the weakest biofilm was formed by the strain isolated from carrots the weakest (Fig. 1).

\section{Discussion}

Biofilm formation by L. monocytogenes is common, but undesirable, especially in the food industry. These strains in the biofilm structure may be a source of secondary food contamination. The conventional disinfection methods are less effective in eliminating this structure. Many factors influence the development of biofilm, including the availability of nutrients, temperature and $\mathrm{pH}$.

In this study, the ability to form biofilm by three L. monocytogenes strains: isolated from human blood, from carrots and the L. monocytogenes ATCC 7644 reference strain, on the polypropylene surface in the presence of increasing addition of sheep blood to the medium was evaluated. The addition of sheep blood in a volume of 5.0, 10.0 and $20.0 \%$ was found to influence the increase in the number of cells recovered from the biofilm for all tested strains. However, the addition of blood in a volume of $50.0 \%$ caused a decrease in biofilm formation in the case of the reference strain and strain isolated from carrots. The highest cell recovery of bacteria from the biofilm for the strain isolated from the human blood has been demonstrated. Di Xia et al. [11] investigated the effect of human plasma on biofilm formation by Streptococcus agalactiae, Streptococcus pyogenes and Staphylococcus aureus. They assessed the impact of $15.0 \%$ human plasma and $1.0 \%$ of glucose in the nutrient medium on the ability to form biofilms. It was shown that all strains of $S$. agalactiae form a biofilm in the broth supplemented with human plasma, while the strains of $S$. aureus and $S$. pyogemes under these conditions do not form a biofilm structure. The authors found that human plasma rich in proteins and fatty acids is used by $S$. agalactiae which results in changes in the composition of bacterial shields [11]. Among other factors influencing the ability of biofilm formation by microorganisms, the presence of glucose and ethanol is mentioned. Sachin et al. [12] showed that the most durable biofilms were formed by strains grown under nutrient-poor conditions, and least intensively - in nutrient-rich medium (cerebral-heart broth). They found that minimal nutritional conditions can stimulate biofilm formation by L. monocytogenes [12].Similar results were obtained by Zhou et al. [9] who showed that the recovery of $L$. monocytogenes from biofilm was the highest in minimal medium as compared to $\mathrm{BHI}$. On the other hand, Pan et al. [13] showed that increase of sodium chloride concentration from $0.5 \%$ to $7.0 \%$ at $22.5^{\circ} \mathrm{C}$ and $30^{\circ} \mathrm{C}$, stimulate $L$. monocytogenes strains to form the biofilm. The optimal concentration of sodium 
chloride was $5.0 \%$ at $22.5^{\circ} \mathrm{C}$. In addition, L. monocytogenes serotype $1 / 2 a$ strains (more often isolated from food products) have been shown to form a biofilm with a higher density than serotype $4 \mathrm{~b}$ strains, with higher glucose concentration and at higher temperature. These researchers also found that the addition of $3.0 \%$ and $5.0 \%$ of ethanol inhibited the formation of biofilms. The biofilm density of the tested strains increased with rising incubation temperature. Temperature, glucose and sodium chloride have a synergistic effect in the biofilm formation by $L$. monocytogenes strains [13]. Limited access to nutrients in minimal media can stimulate stress in L. monocytogenes cells, thereby inducing enhanced biofilm formation. Stress factors include temperature, limited availability of nutrients and increased oxygen availability [5]. In the present study, it was found that the highest cell recovery of $L$. monocytogenes from a biofilm with increasing addition of sheep blood was observed for the strain isolated from the human blood. However, Barbarosa et al. [14] who assessed the ability of biofilm formation by $L$. monocytogenes on the polystyrene under osmotic stress showed that clinical strains were less effective in producing biofilms compared to strains isolated from food [14].

\section{Conclusions}

The addition of sheep blood to the medium at concentrations up to $20.0 \%$ increases the number of cells recovered from the biofilm for all studied $L$. monocytogenes strains whereas medium with $50.0 \%$ blood stimulates the biofilm formation only by the strain isolated from blood. It seems clear that limited availability of nutrients stimulates $L$. monocytogenes to form a biofilm.

Conflict of interest: Authors declare no conflict of interest.

\section{Acknowledgements}

This study was financially supported by the Nicolaus Copernicus University with funds from the maintenance of the research potential of the Department of Microbiology DS-UPB no. 782.

\section{References}

1. Schmid MW, Ng EYW, Lampidis R, et al. Evolutionary history of the genus Listeria and its virulence genes. Syst Appl Microbiol. 2005; 28(1): 1-18, doi: 10.1016/j.syapm.2004.09.005, indexed in Pubmed: 15709360.

2. Jeyaletchumi $P$, Tunung R, Margaret SP, et al. Detection of Listeria monocytogenes in foods. International Food Research Journal. 2010; 17: 1-11.

3. Gandhi M, Chikindas ML. Listeria: A foodborne pathogen that knows how to survive. Int J Food Microbiol. 2007; 113(1): 1-15, doi: 10.1016/j. ijfoodmicro.2006.07.008, indexed in Pubmed: 17010463.

4. Colagiorgi A, Di Ciccio P, Zanardi E, et al. A Look inside the Listeria monocytogenes Biofilms Extracellular Matrix. Microorganisms. 2016; 4(3), doi: 10.3390/microorganisms4030022, indexed in Pubmed: 27681916

5. Srey S, Jahid I, Ha SD. Biofilm formation in food industries: A food safety concern. Food Control. 2013; 31(2): 572-585, doi: 10.1016/j. foodcont.2012.12.001

6. Renier S, Hébraud M, Desvaux M. Molecular biology of surface colonization by Listeria monocytogenes: an additional facet of an opportunistic Gram-positive foodborne pathogen. Environ Microbiol. 2011; 13(4): 835-850, doi: 10.1111/j.1462-2920.2010.02378.x, indexed in Pubmed: 21087384

7. Nowak J, Cruz CD, Palmer J, et al. Biofilm formation of the L. monocytogenes strain $15 \mathrm{G} 01$ is influenced by changes in environmental conditions. J Microbiol Methods. 2015; 119: 189-195, doi: 10.1016/j. mimet.2015.10.022, indexed in Pubmed: 26524221.

8. da Silva EP, De Martinis EC. Current knowledge and perspectives on biofilm formation: the case of Listeria monocytogenes. Appl Microbiol Biotechnol. 2013; 97(3): 957-968, doi: 10.1007/s00253-012-4611-1, indexed in Pubmed: 23233205

9. Zhou Q, Feng X, Zhang Q, et al. Carbon catabolite control is important for Listeria monocytogenes biofilm formation in response to nutrient availability. Curr Microbiol. 2012; 65(1): 35-43, doi: 10.1007/s00284012-0125-4, indexed in Pubmed: 22526570.

10. Van Houdt R, Michiels CW. Biofilm formation and the food industry, a focus on the bacterial outer surface. J Appl Microbiol. 2010; 109(4): 1117-1131, doi: 10.1111/j.1365-2672.2010.04756.x, indexed in Pubmed: 20522145

11. Xia FDi, Mallet A, Caliot E, et al. Capsular polysaccharide of Group B Streptococcus mediates biofilm formation in the presence of human plasma. Microbes Infect. 2015; 17(1): 71-76, doi: 10.1016/j. micinf.2014.10.007, indexed in Pubmed: 25448634.

12. Kadam SR, den Besten HMW, van der Veen S, et al. Diversity assessment of Listeria monocytogenes biofilm formation: impact of growth condition, serotype and strain origin. Int J Food Microbiol. 2013; 165(3): 259-264, doi: 10.1016/j.ijfoodmicro.2013.05.025, indexed in Pubmed: 23800738

13. Pan Y, Breidt F, Gorski L. Synergistic effects of sodium chloride, glucose, and temperature on biofilm formation by Listeria monocytogenes serotype 1/2a and 4b strains. Appl Environ Microbiol. 2010; 76(5): 1433-1441, doi: 10.1128/AEM.02185-09, indexed in Pubmed: 20048067

14. Barbosa J, Borges S, Camilo R, et al. Biofilm Formation among Clinical and Food Isolates of Listeria monocytogenes. Int J Microbiol. 2013; 2013: 524975, doi: 10.1155/2013/524975, indexed in Pubmed: 24489549 